Measurement techniques for melanoma: a statistical comparison

We write in support of the paper by Calder, Campbell, and Plastow. ${ }^{1}$ We have advocated the use of the Vernier scale on grounds of its availability, low cost, and ease of use. ${ }^{2}$ It was therefore pleasing to see that Vernier's invention also provides extremely good reproducibility when compared with the eyepiece graticule or projection image analysis.

One thing the authors omit is the limit of accuracy of the standard stage Vernier scale. This often has 10 Vernier divisions equal to 9 $\mathrm{mm}$ on the fixed scale. The accuracy limit is the difference between one division on each scale: $1 \cdot 0-0.9=0.1 \mathrm{~mm} .^{3}$ If greater accuracy were required it would be easy to replace the Vernier scales for one with smaller divisions, such as one with 25 divisions equal to 24 on the fixed scale. (Electronic Vernier scales now exist taking accuracy to five decimal places). BF WARREN

Department of Histopathology, Bristol Royal Infirmary Marlborough Street, Bristol BS2 $8 H W$ JD DAVIES Regional Breast Pathology Unit,
Southmead Hospital, Bristol BS10 5NB

1 Calder CJ, Campbell AP, Plastow SR. Measurement techniques for melanoma: a statistical comparison. J Clin Pathol 1990 43:922-3.

2 Warren BF, Davies JD. Pierre Vernier's invention: a forgotten tool of our trade. Histopathology (in press)

3 Leeson AE, Sampson P, West P. Practical fitting and workshop technology. London: Pitman 1971:70-1.

\section{Identification of Haemophilus} influenzae

Murphy et al described a comparative study of several commercially available systems for the identification of Haemophilus influenzae. ${ }^{1}$ While the kits tested were found to be satisfactory for identification of the species, the authors concluded that the Rapid NH and RIM systems were not suitable for biotyping when compared with a modification of the APIIOS product. Since Kilian described the application of biotyping to $H$ influenzae, based on three biochemical properties, ${ }^{2}$ however, data accumulated on the prevalence and distribution of organisms of each biotype have indicated that this typing system is not generally useful for epidemiological purposes.

Apart from the more unusual and the rare biotypes (IV-VIII), biotyping of $H$ influenzae is not so discriminative that it can provide even presumptive evidence that two or more isolates are identical. For example, more than $80 \%$ of capsule type b isolates, which are responsible for most invasive infections due to $H$ influenzae, are of biotype $\mathrm{I}{ }^{34}$ Among the 2434 Hinfluenzae collected from England and Scotland in 1986, 82 of the total 87 encapsulated organisms and 57 of 66 type b isolates were of biotype $I,{ }^{5}$ confirming that biotyping is not likely to be of use in establishing the epidemiology of $H$ influenzae meningitis or epiglottitis.

Moreover, data from the same United Kingdom study showed that $33 \%$ of 2042 isolates from the respiratory tract were of biotype II and $30 \%$ were biotype III, while isolates from eyes were very predominantly of these two biotypes $(46 \%$ and $43 \%$, respectively, of 273 isolates). Two national studies have also reported that antimicrobial resistance does not seem to have any correlation with biotype. ${ }^{56}$ Biotyping, therefore, does not seem to be a useful epidemiological tool in the study of spread of isolates in community or hospital populations.

On the basis of these results, it seems unlikely that routine biotyping of $H$ influenzae in diagnostic laboratories would provide any useful information to clinical microbiologists, epidemiologists, or physicians. There are, however, several techniques in use in centres with a research interest in this species which discriminate well between isolates. Outer membrane protein subtyping and multilocus enzyme electrophoresis, used alone, and preferentially in combination, ${ }^{47}$ have provided very useful data on worldwide and local distribution of groups of type $b$ and noncapsulate $H$ influenzae.

M POWELL Department of Medical Microbiology, The London Hospital Medical College, Turner Street, London E1 $2 A D$

1 Murphy PG, Craig I, Lafong AC, Smyth ETM. Evaluation of two rapid methods for identifying and biotyping Haemophilus influenzae. $J$ Clin Pathol 1990;43:581-3.

2 Kilian M. A rapid method for the differentiation of Haemophilus strains. Acta Pathol Microbiol Immunol Scand 1974;82:835-42.

3 Long SS, Teter MJ, Gilligan PH. Biotype of Haemophilus influenzae: correlation with virulence and ampicillin resistance. $J$ Infect Dis 1983;147:800-6.

4 Barenkamp SJ, Munson RS, GranoffDM. Comparison of outer-membrane protein subtypes and biotypes of isolates of Haemophilus influenzae. J Infect Dis 1983;144:480.

5 Powell M, Koutsia-Carouzou C, Voutsinas D, Seymour A, Williams JD. Resistance of clinical isolates of Haemophilus influenzae in United Kingdom 1986. Br Med J 1987; 295:176-9.

6 Dabernat H, Delmas C, Lareng MB. Prévalence de la résistance aux antibiotiques des Haemode la resistance aux antibiotiques des Haemophilus influenzae isolés

7 Musser JM, Barenkamp SJ, GranoffDM, Selander RK. Genetic Relationships of serologically non-typable and serotype $b$ strains of Haemophilus influenzae. Infect Immun 1986; 52:183-91.

Drs Murphy, Craig, La Fong, and Smyth comment:

We welcome Dr Powell's clarifying comments on the usefulness of Kilian biotyping of $H$ influenzae. Our findings of $35 \%$ biotype I among isolates in Northern Ireland make biotyping of slightly greater value in this population than Dr Powell's data suggest.' In the Northern Ireland collection we also reported on a higher incidence of ampicillin resistance than that reported in the England/ Scotland study, so we are clearly dealing with a different population. ${ }^{2}$

The biotyping element of our study was included simply because the data were produced as an intricate part of identification kits as marketed by the manufacturers. $H$ influenzae biotyping has also never been reported before in Northern Irish isolates. While we would most certainly not suggest "routine biotyping of $H$ influenzae in diagnostic laboratories", nevertheless, if these kits were being used for identification it seems pointless to ignore freely available biotyping data if it were relevant.

We also agree wholeheartedly with $\mathrm{Dr}$ Powell's comments on the general usefulness of multilocus enzyme electrophoresis as we have extensive experience of this genotypic method of clonal analysis which not only generates data for epidemiological application, as Dr Powell suggests, but also produces genetic diversity data that are useful in the systematics of the taxon.

1 Powell M, Koutsia-Carouzou C, Voutsinas D, Seymour A, Williams JD. Resistance of clinical isolates of Haemophilus influenzae in United Kingdom. $1986 \mathrm{Br}$ Med $J$ 1987; 295:176-9.

2 Murphy PG, Craig I, La Fong C. The prevalence of antibiotic resistance in Haemophilus influenzae in Ireland. $J$ Antimicrob Chemother 1990;25:1026-6.

Vulvo-vaginal irritation in diabetic women

We congratulate Rowe $e t$ al on their study of the cause of vulvo-vaginal irritation in women with diabetes mellitus. ${ }^{1}$ It is essential that such research into the accuracy of established clinical dogma is performed. We are concerned, however, that the methods used in this study may be incomplete and may have led to a misleading conclusion.

Firstly, the method of obtaining vaginal samples was not clearly specified. Failure to use a speculum to provide direct access to the vaginal walls is an error which not only may result in contamination with microbiological flora from the skin or bowel, or both, but may also hinder thorough clinical examination.

Secondly, in genitourinary medicine clinics it is usual to examine microscopically a Gram stained vaginal smear, as well as take a culture, to diagnose vaginal candidiasis. We have found that $56 \%$ of women with vaginal discharge use "self-medication" before attending our clinic-26\% with topical antifungal medications. In this latter group, microscopy of a Gram stained smear was frequently positive for Candida spores and hyphae while culture on Sabourand's media was negative. ${ }^{2}$ In Rowe's study one quarter of the patients had previously been prescribed antifungal medications, but no mention is made as to when they were last applied. We believe a full drug and topical medication history is important and that microscopy should have been included in the assessment of these women.

Thirdly, no attempt was made to diagnose bacterial vaginosis; a $\mathrm{pH}$ test and amine test should have been performed.

Although a surprisingly small number of the women studied were sexually active, we do think that screening for sexually acquired infections (gonorrhoea, chlamydia) should have been performed. A high vaginal swab does not exclude Trichomonas vaginalis; an important cause of vulvo-vaginitis which was ignored in this study.

The low prevalence of sexual activity may in itself be due to problems that could benefit from intervention. Chronic vulvo-vaginal pathology, either infected or postmenopausal, may be associated with psychosexual dysfunction. Perhaps sensitive questioning in this area and careful examination may highlight problems which could be solved.

Finally, many of the organisms, other than Candida, cultured by Rowe et al are considered opportunistic invaders of abnormal vaginal epithelium. ${ }^{4}$ Defects of immune function do occur in diabetic patients, particularly those affecting leucocyte migration function, 